\title{
The study on the Mal-operation of the transformer current differential protection during external faults cause by CT saturation
}

\author{
Jianhong $\mathrm{Wu}^{1}$, Xuanwei $\mathrm{Qi}^{2, *}$, Wang Song ${ }^{2}$, Wenbin $\mathrm{Cao}^{2}$, Jun $\mathrm{He}^{1}$, and Lan $\mathrm{Mao}^{3}$ \\ ${ }^{1}$ E. Energy Technology Co., Ltd., Hangzhou, Zhejiang Province, China \\ ${ }^{2}$ State Grid Zhejiang Electrical Power Research Institute, Hangzhou, Zhejiang Province, China \\ ${ }^{3}$ Beijing In-To Simulation Technology Ltd., Beijing, China
}

\begin{abstract}
This paper analyses the unusual mal-operation of the differential protection for transformer current differential protection during the external fault. The mal-operation reason is researched in detail, based on the mathematical analysis, the simulation, and the field recorded fault data analysis. It is disclosed that the CT saturation leads to the maloperation. Based on this, this paper analyses the improvement for the CT saturation identification method, and discusses the CT selection principles. This paper is aimed at attracting the attention on the problem of the CT saturation and proposing the reference of the analysis method the countermeasure for this problem.
\end{abstract}

Keywords: CT saturation; Current differential protection; Transformer

\section{Introduction}

Recently, several transformer differential current protection mal-operate, which is caused by the CT saturation during the external fault, and the running transformers are forced to be out of service, which threatens the safe and sound operation of the power grid and power equipment[1-10]. This paper analyses this sort of mal-operation problem.

The transient process of CT saturation is researched through mathematical analysis, the simulation study and the on-site fault record study. The electromagnetic process analytical expression is solved based on the CT model. The precise Jiles-Atherton CT simulation model is established, and the saturation process is replayed buy the simulation approach.

It is disclosed that the CT saturation leads to the mal-operation. Based on this, this paper analyses the improvement for the CT saturation identification method, and discusses the CT selection principles. The on-site fault record data proves the validity of the simulation results. Based on this, the relevant countermeasure for the protection is proposed, and the selection method of CT type is discussed.

In this paper, the mal-operation case is introduced in Section2. The mathematical mechanism of CT saturation process is analysed in section 3. The digital simulation research is carried out in Section 4. The relevant improvement method is introduced in Section 5. In the end, this research is concluded in Section 6.

\footnotetext{
*Corresponding author: 814512663@qq.com
} 


\section{The Mal-operation Case}

The capacity of a $\mathrm{Y} / \mathrm{Y} / \triangle 11$ transformer is $180 \mathrm{MVA}$, and its nominal voltage is $230 \mathrm{kV} / 117 \mathrm{kV} / 35 \mathrm{kV}$. The differential mal-operates during the external fault at the $35 \mathrm{kV}$ side out of the transformer,.

As shown in Fig.1, during the external fault, the large amplitude fault current flows through the transformer, which has the long-delay DC component. The fundamental frequency component of the fault current is about 5 6 times of the normal current ( $2 \mathrm{kA})$ at $35 \mathrm{kV}$ side. From the time of $0.27 \mathrm{~s}$, the phase A CT is saturated, causing the distorting of the secondary current. As a result, the false differential current of the transformer increases, as can be seen in Fig.2. The transformer current differential protection has the second harmonic restrict criterion. The second harmonic component is shown in Fig.3. During the period between $0.04 \mathrm{~s} \sim 0.05 \mathrm{~s}$, the second harmonic component is less than the set threshold $15 \%$. The differential current and the restraint current located in the operation region in the restraint plane. Therefore, the transformer differential protection mal-operates.

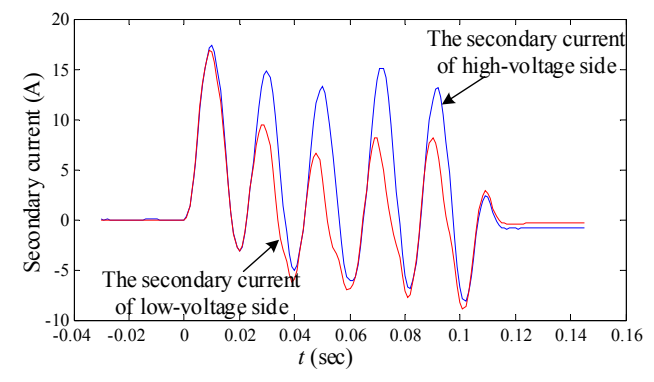

Fig. 1. The transformer currents during the mal-operation

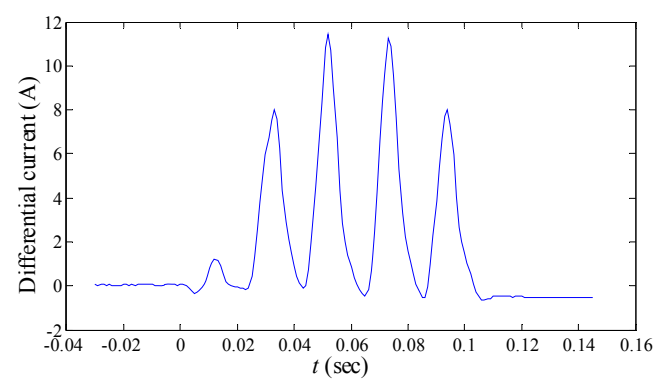

Fig. 2. The differential currents of the transformer

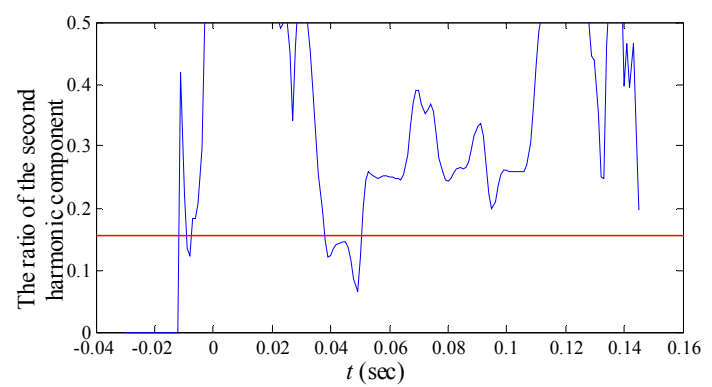

Fig. 3. The harmonic component of the differential currents of the transformer 


\section{Mathematical Analysis of CT Saturation Process}

In order to discover the $\mathrm{CT}$ saturation mechanism in depth, the mathematical analysis of CT saturation process is carried out. The fault current is modelled by the fundamental component with the long-lasting DC component. Based on the equipment circuit of CT and the input primary current. The flux of the iron core can be solved. Comparing the calculated flux and the saturated flux, the time when the CT saturated can be obtained.

The primary current can be fitted by the expression (1). The curve of expression (1) is the same as the actual fault current, as compared in Fig.4.

$$
i_{\mathrm{p}}(t)=\sqrt{2} I_{\mathrm{psc}}\left[\mathrm{e}^{-t / T_{\mathrm{p}}} \cos \theta-\cos (\omega t+\theta)\right] \mathrm{A}=\sqrt{2} \times 14000 \times\left[\mathrm{e}^{-t / 0.05} \cos \left(-5^{\circ}\right)-\cos \left(\omega t-5^{\circ}\right)\right] \mathrm{A}
$$

Where, $I_{\mathrm{psc}}$ is the amplitude of the fundamental frequency current, $T_{\mathrm{P}}$ is the decaying time constant of the dc component, $\theta$ is the phase of the fundamental frequency current at the fault inception.

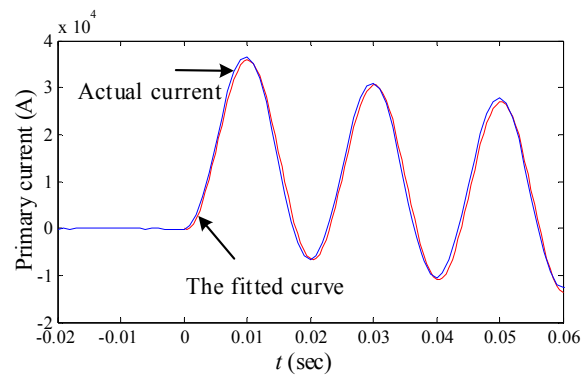

Fig. 4. The comparison curve of the fitted primary current

The parameters of the saturated CT is tested by OMICROM, as shown in Table 1. The equivalent circuit of CT is shown in Fig.5. The expression of flux can be obtained as (2). It is known that the saturation flux of CT is $3.0559 \times 10^{-3} \mathrm{~Wb}$. The calculated flux in the timedomain is shown in Fig.6. The CT flux will increase higher than the saturation point at $0.026 \mathrm{~s}$. According to the fault recording data, the CT saturates at 0.26 after the fault inception.

The mathematical analysis is accord with the actual situation. The results indicates that the DC component from the primary fault current will drive the bias flux in the CT iron core and will drive CT into saturation.

$$
\begin{aligned}
& \phi=\frac{\sqrt{2} I_{\mathrm{psc}} T_{\mathrm{s}} R_{\mathrm{s}}}{K_{\mathrm{n}} N_{\mathrm{s}}}\left[\frac{T_{\mathrm{p}}}{T_{\mathrm{p}}-T_{\mathrm{s}}}\left(\mathrm{e}^{-\frac{t}{T_{\mathrm{p}}}}-\mathrm{e}^{-\frac{t}{T_{\mathrm{s}}}}\right) \cos \theta+\frac{\sin \theta \mathrm{e}^{-\frac{t}{T_{\mathrm{s}}}}}{\omega T_{\mathrm{s}}}-\frac{\sin (\omega t+\theta)}{\omega T_{\mathrm{s}}}\right] \\
& =\left[-0.01032 \times \mathrm{e}^{-\frac{t}{0.05}}+0.01026 \times \mathrm{e}^{-\frac{t}{7.57}}-0.0006552 \sin \left(100 \pi \times t-5^{\circ}\right)\right] \mathrm{A}
\end{aligned}
$$

Where, $K_{\mathrm{n}}$ is the ratio of CT, $N_{\mathrm{s}}$ is the number of windings, $T_{\mathrm{s}}$ and $R_{\mathrm{s}}$ are the time constant and the resistance of the secondary loop of CT respectively. 
Table 1. The test results of the CT parameters

\begin{tabular}{llll}
\hline Ratio $\left(K_{\mathrm{n}}\right)$ & $\begin{array}{l}\text { The number of } \\
\text { windings }\left(N_{\mathrm{s}}\right)\end{array}$ & $\begin{array}{l}\text { The un-saturated } \\
\text { inductance }\left(L_{\mathrm{e}} / \mathrm{H}\right)\end{array}$ & $\begin{array}{l}\text { The resistance of the } \\
\text { secondary windings } \\
\left(R_{\mathrm{ct}} / \Omega\right)\end{array}$ \\
\hline 400 & 400 & 12.596 & 0.863 \\
\hline $\begin{array}{l}\text { The rated } \\
\text { burden } \\
\text { resistance } \\
\left(R_{\mathrm{bn}} / \Omega\right)\end{array}$ & $\begin{array}{l}\text { The rated time } \\
\text { constant of } \\
\text { secondary loop } \\
\left(T_{\mathrm{sn}} / \mathrm{s}\right)\end{array}$ & $\begin{array}{l}\text { The actual burden } \\
\text { resistance } \\
\left(R_{\mathrm{b}} / \Omega\right)\end{array}$ & $\begin{array}{l}\text { The acutual time } \\
\text { constant of secondary } \\
\text { loop } \\
\left(T_{\mathrm{s}} / \mathrm{s}\right)\end{array}$ \\
\hline 1.6 & 5.1 & 0.6 & 8.585 \\
\hline
\end{tabular}

Fig. 5. The equivalent diagram of the CT circuit

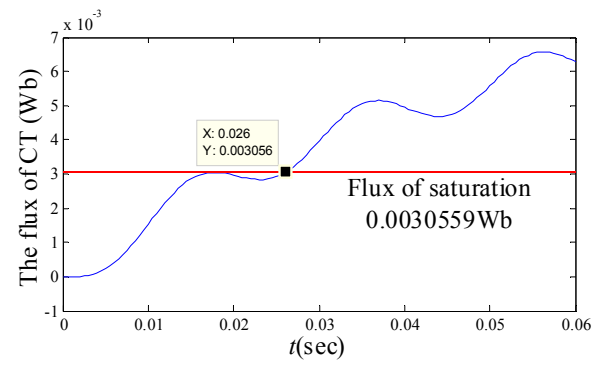

Fig.6. The calculated CT flux

\section{Digital Simulation Research}

The Jiles-Atherton CT model is established by the electromagnetic transient software ${ }^{[12]}$, in order to research the maloperation case. The excitation characteristic of the on-site CT in Case One is fitted by the Jiles-Atherton model, as shown in Fig.7. The parameters of the Jiles-Atherton model is fitted by the Simulated genetic algorithm ${ }^{[13]}$. The comparison curves of the excitation characteristic in Fig.7 indicates that the simulated curve and the model actual measured is highly identical. The residual flux is set as $51 \%$ according to the actual situation, and the hysteresis loop is shown in Fig.8.

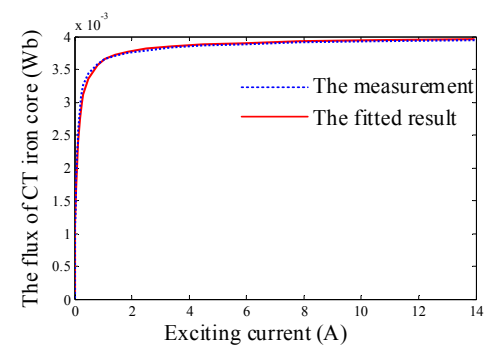

Fig. 7. The exciting characteristic curves of CT 

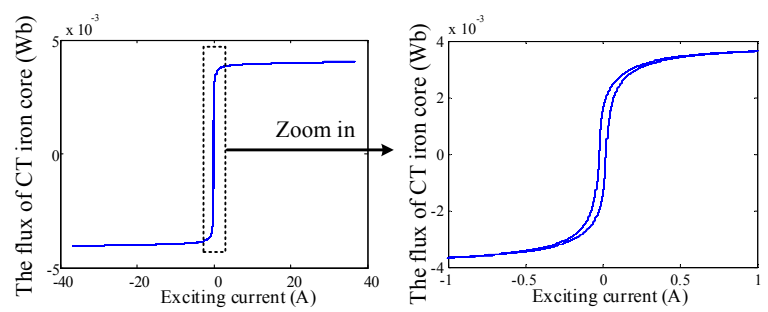

Fig. 8. The hysteresis loop of CT

The comparison of the actual and the simulated saturated CT secondary current curves are illustrated in Fig.9. The simulated curve is highly accord with the on-site measured curve. The simulated hysteresis loop is shown in Fig.10. It is obvious that the flux of the CT iron core accumulates the bias during the external fault. Hence, the CT saturation occurs and the false differential current increases.

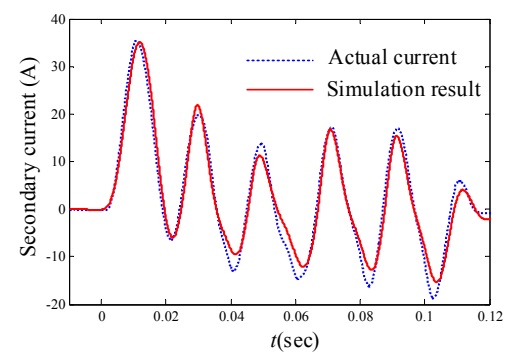

Fig. 9. The simulation result of CT saturation
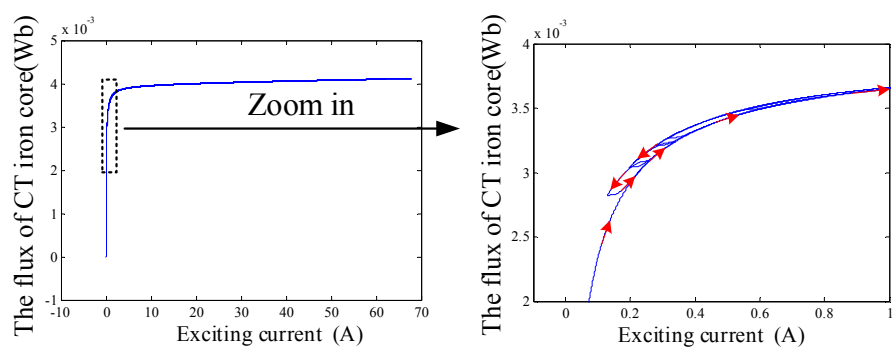

Fig. 10. The CT hysteresis loop simulation result

\section{The Improvement}

According to the typical characteristic of the protective CT, the CT can linearly transfer the primary current during initial $3 \sim 5 \mathrm{~ms}$ post-fault moment. Because the CT needs time to accumulate the bias flux before saturated.

As shown in Fig.1, the CT comes into saturation at $0.27 \mathrm{~s}$ respectively after the external fault inception. The restraint current will raise significantly when the external fault occurs. However, the false differential current will raise later by the CT saturation. Hence, there exists a time span between the moments when the restraint current and the differential current increase.

The trace of the differential/restraint current in the restraint plane for the mal-operation case is shown in Fig.11. At the initial moment after the fault inception, the restraint current increase while the differential current still remain low value because the CT has not come 
into saturation. Hence, the trace move in the right direction. When the CT comes into saturation later, the trace move up into the operation zone. The additional restraint zone in the restraint plane can recognize this sort of false differential current, and can prevent the mal-operation.

The differential current and the restraint current will increase synchronously during the internal fault. The differential/restraint current will fast come into the operation zone directly, rather than passing through additional restraint zone. Hence, the method based on the additional restraint zone can both guarantee the reliability during the external fault and the sensitivity during the internal fault.

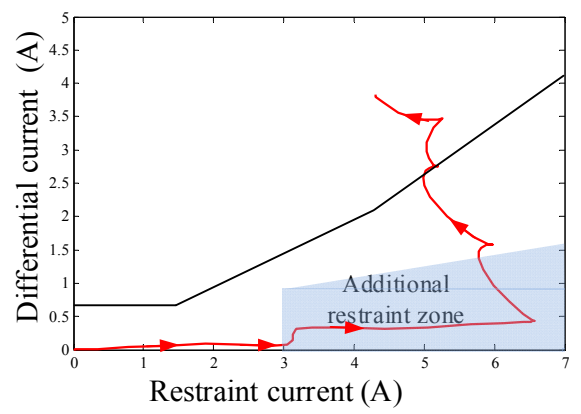

Fig. 11. The add-on stabilization (CASE One)

\section{Conclusion}

In this paper, through theoretical analysis, digital simulation, and analysis of on-site recorded data, in-depth research is carried out. The research results show that during the occurrence of short-circuit faults, P-type CTs are transiently saturated due to the attenuated non-periodic components in the primary current, and the false differential current increases. The differential protection lacks the identification criterion for CT saturation. Therefore, the differential protection of transformer occurs. The saturation recognition criterion of CT based on the time difference method and additional restraint zone method can effectively avoid this sort of mal-operation.

Current transformer is the key link for relay protection device to obtain grid information, and its transfer characteristics will directly affect the performance of protection. As the power grid gradually develops into a large AC/DC hybrid power grid, the short-circuit capacity of the system is gradually increasing, and the electromagnetic transient process is becoming more and more complicated. How to select CTs scientifically and rationally with consideration of cost and performance is a key issue that needs to be considered in power construction.

This paper introduces the analysis methods and improvement strategies for the transient saturation of transformers, and discusses various factors that need to be considered in the selection of transformers, hoping to provide references for related scholars and experts.

\section{Acknowledgement}

This paper was founded by the science \& technology project of E. Energy Technology Co., Ltd: HZYN-2019-0122-308. 


\section{References}

1. X Xiaochun, L Geming, D Guangwu,et al. Energy Re-sistance Based Anti-saturation Scheme with Break-er-and-a-half Configuration[J]. Automation of Electric Power Systems,2016, 40(12): 189-193, 215(in Chinese).

2. Q Xuan-wei, Y Xiang-gen, Z Zhe,et al. The Study on the Mal-operation Reason for the Current Differential Protec-tion due to the Adjacent Transformer Switching-on and Its Countermeasure[J]. Automation of Electric Power Sys-tems,2016, 40(03): 129-134(in Chinese).

3. Z Jiankang, S Xiaohua, L Huaiqiang,et al. Impact of Large-capacity SVC on Transformer Differential Protec-tion and the Solutions[J]. Automation of Electric Power Systems,2015, 39(7): 164-168(in Chinese).

4. Y Jiang, Z Hongyang, C Zhaohui,et al. Improvement of 500kV Breaker Failureand Dead-zone Prevention Based on Requirement on Stability of Grid[J]. Automation of Electric Power Systems,2015, 39(2): 142-146(in Chi-nese).

5. W Yi, Q Xuanwei, L Hang,et al. Complex Sympathetic Inrush and Its Influence on Current Differential Protec-tion[J]. Automation of Electric Power Systems, 2014, 38(6): 98-105(in Chinese).

6. S Xin, Z Yongli, S Churila,et al. Power transformer fault classifying model based on deep belief network[J]. Power System Protection and Control,2016, 44(1): 71-76(in Chinese).

7. F Wei, Z Lihua, L Yong,et al. Study on no-load closing inrush current of transformer group and suppression measures[J]. Power System Protection and Con-trol,2015,1): 28-33(in Chinese).

8. S Wenquan, Q Ni, W Jianbo. A novel algorithm of iden-tifying inrush current based on waveform cross-correlation coefficient[J]. Power System Protection and Control,2015,23): 14-20(in Chinese).

9. L Sheng, Z Xiaofan. A transformer differential protection method based on multicriterion[J]. Power System Protec-tion and Control,2015,23): 125-131(in Chinese).

10. C Yunfeng, X Yang, P Qi. PSCAD simulation of trans-former longitudinal differential protection based on custom model[J]. Power System Protection and Control,2015,3): 118-122(in Chinese).

11. Annakkage U D, McLaren P G, Dirks E,et al. A current transformer model based on the Jiles-Atherton theory of ferromagnetic hysteresis[J]. Power Delivery, IEEE Transactions on, 2000, 15(1):57-61.

12. Q Xuanwei, Y Xianggen, L Gan,et al. A construction method for the simulation platform for the analysis of the current transformer[J]. Power System Protection and Control,2015, 43(22): 69-76(in Chinese).

13. Xuanwei Q, Xianggen Y, Zhe Z,et al. Study on the Unu-sual Misoperation of Differential Protection during Transformer Energization and Its Countermeasure[J]. IEEE Transactions on Power Delivery, 2016, 31(5):1998-2007. 